PROCEEDINGS OF THE

AMERICAN MATHEMATICAL SOCIETY

Volume 125, Number 10, October 1997, Pages 2891-2896

S 0002-9939(97)03953-1

\title{
PROPERTY (M) AND THE WEAK FIXED POINT PROPERTY
}

\author{
JESÚS GARCIA FALSET AND BRAILEY SIMS
}

(Communicated by Palle E. T. Jorgensen)

\begin{abstract}
It is shown that in Banach spaces with the property (M) of Kalton, nonexpansive self mappings of nonempty weakly compact convex sets necessarily have fixed points. The stability of this conclusion under renormings is examined and conditions for such spaces to have weak normal structure are considered.
\end{abstract}

\section{INTRODUCTION}

Throughout $X$ will denote a Banach space, $B_{X}$ its unit ball $\{x \in X:\|x\| \leq 1\}$, $S_{X}$ its unit sphere $\{x \in X:\|x\|=1\}$, and $X^{*}$ the dual space of $X$.

A weakly null type on a Banach space $X$ is a function of the form

$$
\psi_{\left(x_{n}\right)}(x)=\limsup _{n}\left\|x-x_{n}\right\|,
$$

where $\left(x_{n}\right)$ is a weak null sequence. We say $\psi_{\left(x_{n}\right)}$ is nontrivial if $\psi_{\left(x_{n}\right)}(0) \neq 0$; that is, if $\left\|x_{n}\right\| \not \rightarrow$. If $X$ is separable we may replace $\left(x_{n}\right)$ by a subsequence so that $\psi_{\left(x_{n}\right)}(x)=\lim _{n}\left\|x-x_{n}\right\|$, for all $x \in X$.

Over the last decade an intimate connection has been established between the structure of certain weak null types and the geometry of the space, in particular the presence of weak normal structure, or the weak fixed point property. See, for example, Maurey [9], and Sims [12].

A Banach space $X$ has weak normal structure if there are no nontrivial weakly compact convex diametral subsets. That is, if $C$ is a weakly compact convex subset with $\operatorname{diam} C>0$ then $\inf _{y \in C} \sup _{x \in C}\|y-x\|<\operatorname{diam} C$.

Weak normal structure is a sufficient condition for the weak fixed point property (w-fpp): Every nonexpansive self mapping of a nonempty weakly compact convex subset of $X$ has a fixed point. Here $T: C \rightarrow C$ nonexpansive means $\|T x-T y\| \leq$ $\|x-y\|$, for all $x, y \in C$.

The reader is referred to the book by Goebel and Kirk [3] for a full discussion of these properties and the connection between them.

Recently Kalton [5] introduced property $(M)$ : Weakly null types are constant on spheres about the origin. That is, for $x_{n} \stackrel{w}{\longrightarrow} 0$ the weakly null type $\psi_{\left(x_{n}\right)}(x)=$ $\lim \sup _{n}\left\|x-x_{n}\right\|$ is a function of $\|x\|$ only.

Received by the editors January 3, 1996 and, in revised form, April 19, 1996.

1991 Mathematics Subject Classification. Primary 47H09, 47H10, 46B20.

Partially supported by grant DGICYT PB-1177-c02-02 and a travel grant from the University of Newcastle.

(C) 1997 American Mathematical Society 
Property (M) was an essential ingredient in Kalton's characterization of those separable Banach spaces $X$ for which the compact operators $\mathcal{K}(X)$ form an M-ideal in the algebra of all bounded linear operators, $\mathcal{L}(X)$. That is,

$$
\mathcal{L}(X)^{*}=\left(\mathcal{K}(X)^{\perp} \oplus V\right)_{1}, \quad \text { for some closed subspace } \mathrm{V} .
$$

A link with the fixed point property for such spaces was observed in Sims [10] where it was noted that Åsvald Lima [7] had effectively shown that $\mathcal{K}(X)$ an M-ideal in $\mathcal{L}(X)$ implies that $X^{*}$ is weak*-uniformly Kadec-Klee $\left(\mathrm{UKK}^{*}\right)$ and hence has weak* normal structure [1]. It therefore seems natural to inquire into connections between property $(\mathrm{M})$, weak normal structure, and the $\mathrm{w}-\mathrm{fpp}$. This is particularly relevant since a detailed study of spaces with property (M), including stability under sums and renormings, has been undertaken by Kalton [5] and Kalton and Werner [6], to which the reader is referred for details and examples.

\section{Property (M) And Weak normal structure}

Property $(\mathrm{M})$ relates to several properties previously introduced in connection with the $\mathrm{w}$-fpp.

A Banach space $X$ is said to have WORTH if every weakly null type satisfies $\psi_{\left(x_{n}\right)}(-x)=\psi_{\left(x_{n}\right)}(x)$, for all $x \in X[11]$, and to satisfy the non-strict Opial condition if every weakly null type satisfies $\psi_{\left(x_{n}\right)}(0) \leq \psi_{\left(x_{n}\right)}(x)$, for all $x \in X$.

Proposition 2.1. For the following conditions on the Banach space $X$ we have (i) $\Longrightarrow$ (ii) $\Longrightarrow$ (iii) $\Longrightarrow$ (iv).

(i) X has property (M).

(ii) X has WORTH.

(iii) If $x_{n} \stackrel{w}{\longrightarrow} 0$ then for each $x \in X$ we have $\psi_{\left(x_{n}\right)}(t x)$ is an increasing function of $t$ on $[0, \infty)$.

(iv) $X$ satisfies the non-strict Opial condition.

Proof. All the implication are clear except for (ii) $\Longrightarrow$ (iii). To see this, note that for $0<t_{1}<t_{2}$ there exists $\beta \in(0,1)$ such that $t_{1} x=\beta\left(-t_{2}\right) x+(1-\beta) t_{2} x$ and so, since $\psi_{\left(x_{n}\right)}$ is convex and by WORTH $\psi_{\left(x_{n}\right)}\left(-t_{2} x\right)=\psi_{\left(x_{n}\right)}\left(t_{2} x\right)$, we have $\psi_{\left(x_{n}\right)}\left(t_{1} x\right) \leq \beta \psi_{\left(x_{n}\right)}\left(-t_{2} x\right)+(1-\beta) \psi_{\left(x_{n}\right)}\left(t_{2} x\right)=\psi_{\left(x_{n}\right)}\left(t_{2} x\right)$.

An immediate consequence is Lemma 2.1(3) of Kalton [5].

Corollary 2.2. If $X$ has property $(M)$ any weakly null type $\psi_{\left(x_{n}\right)}(x)$ is an increasing function of $\|x\|$.

It is well known (see, for example, [3]) that if $X$ fails to have weak normal structure then $B_{X}$ contains a weak null sequence $\left(x_{n}\right)$ satisfying

$$
\lim _{n}\left\|x-x_{n}\right\|=1, \quad \text { for all } x \in \overline{\mathrm{co}}\left\{x_{n}\right\}_{n=1}^{\infty} .
$$

In particular, since $0 \in \overline{\mathrm{co}}\left\{x_{k}\right\}_{k=1}^{\infty}$, we have $\left\|x_{n}\right\| \longrightarrow 1$. Thus we have the following.

Proposition 2.3. Let $X$ be a Banach space with property (M). If $X$ fails to have weak normal structure then $X$ admits a nontrivial weakly null type which is identically equal to 1 on $B_{X}$.

Proof. Let $\left(x_{n}\right)$ be a sequence in $B_{X}$ such as described in the previous paragraph. Then $\psi_{\left(x_{n}\right)}(0)=1$ and $\psi_{\left(x_{n}\right)}\left(x_{m}\right)=1$, for all $m$. Since $\left\|x_{m}\right\| \rightarrow 1$, it follows from Corollary 2.2 and property $(\mathrm{M})$ that $\psi_{\left(x_{n}\right)}$ equals 1 on the open unit ball, and hence by continuity on $B_{X}$. 
Theorem 2.4. Let $X$ be a Banach space with property $(M)$. Then $X$ has weak normal structure if there exists a point $x_{0} \in S_{X}$ such that whenever $y_{n} \stackrel{w}{\longrightarrow} x_{0}$ and $\left\|y_{n}\right\| \longrightarrow 1$ we have that the separation index $\gamma\left(y_{n}\right):=\operatorname{sup~inf}_{k \neq m}\left\|y_{n_{k}}-y_{n_{m}}\right\|<1$, where the supremum is taken over all subsequences $\left(y_{n_{k}}\right)$ of $\left(y_{n}\right)$.

Proof. Suppose $X$ fails to have weak normal structure. Let $\psi_{\left(x_{n}\right)}$ be the weakly null type of Proposition 2.3. and let $y_{n}:=x_{0}-x_{n}$. Then, $y_{n} \stackrel{w}{\rightarrow} x_{0}$ and $\lim \sup _{n}\left\|y_{n}\right\|=$ $\psi_{\left(x_{n}\right)}\left(x_{0}\right)=1$, so there is a subsequence with $\gamma\left(y_{n_{k}}\right)<1$. But, $\gamma\left(y_{n_{k}}\right)=\gamma\left(x_{n_{k}}\right)=1$, a contradiction.

Corollary 2.5. If $X$ has property $(M)$ and satisfies any of the following then $X$ has weak normal structure.

(i) $X$ has the Kadec-Klee property (the relative weak and norm topologies agree on $S_{X}$ ).

(ii) $X$ is reflexive.

(iii) $X$ has the Radon-Nikodym property.

(iv) $X$ has the point of continuity property: for every weakly closed bounded subset $A$, the identity map $(A$, weak) to $(A$, norm $)$ has at least one point of continuity; see [2] for details.

(v) $S_{X}$ contains at least one point at which the relative weak and norm topologies agree.

Proof. (i) $\Longrightarrow$ (v), (ii) $\Longrightarrow$ (iii) $\Longrightarrow$ (iv) $\Longrightarrow(v)$, and (v) implies the condition of Theorem 2.4 .

\section{Property (M) implies the Weak fiXed point property}

For a weakly null type $\psi_{\left(x_{n}\right)}$ on the Banach space $X$ define

$$
\lambda_{\left(x_{n}\right)}:=\sup \psi_{\left(x_{n}\right)}\left(B_{X}\right) \text {. }
$$

From the weak lower semi-continuity of the norm, we have

$$
1 \leq \lambda_{\left(x_{n}\right)} \leq 1+\limsup _{n}\left\|x_{n}\right\|,
$$

and if $X$ has property $(\mathrm{M})$ then

$$
\begin{aligned}
\lambda_{\left(x_{n}\right)} & =\psi_{\left(x_{n}\right)}(x), \quad \text { for any } x \in S_{X}, \\
& =\lim _{m} \psi_{\left(x_{n}\right)}\left(y_{m}\right), \quad \text { whenever }\left\|y_{m}\right\| \longrightarrow 1 .
\end{aligned}
$$

Lemma 3.1. If $X$ has property $(M)$ and $\psi_{\left(x_{n}\right)}$ is a weakly null type with $\psi_{\left(x_{n}\right)}(0)=$ 1 then $\lambda_{\left(x_{n}\right)}=D\left(x_{n}\right)$, where $D\left(x_{n}\right):=\lim \sup _{m} \limsup _{n}\left\|x_{m}-x_{n}\right\|$.

Proof. $\psi_{\left(x_{n}\right)}(0)=1$ implies that the limsup of the norms of any subsequence of $\left(x_{n}\right)$ is at most 1 , and that there exists a subsequence $\left(x_{n_{k}}\right)$ with $\left\|x_{n_{k}}\right\| \rightarrow 1$.

Then

$$
\begin{aligned}
D\left(x_{n}\right) \leq \lambda_{\left(x_{n}\right)} & =\lim _{k} \psi_{\left(x_{n}\right)}\left(x_{n_{k}}\right) \\
& =\lim _{k} \limsup _{n}\left\|x_{n_{k}}-x_{n}\right\| \\
& \leq D\left(x_{n}\right) .
\end{aligned}
$$


Theorem 3.2. Let $X_{0}:=\left(X,\|\cdot\|_{0}\right)$ be a Banach space having property (M) and let $\|\cdot\|_{1}$ be an equivalent norm on $X$ satisfying

$$
\|x\|_{0} \leq\|x\|_{1} \leq b\|x\|_{0}, \quad \text { for all } x \in X .
$$

If $b<(1+\sqrt{5}) / 2$, then $X_{1}:=\left(X,\|\cdot\|_{1}\right)$ has the $w$-fpp.

Proof. Suppose $X_{1}$ fails the ${ }^{\mathrm{w}}$-fpp. Then, by standard arguments (see [3], for example), there exists a weak compact convex subset $K$ of $X$ with $\operatorname{diam}_{1} K=1$ and a fixed point free $\|\cdot\|_{1}$-nonexpansive map $T: K \rightarrow K$ with respect to which $K$ is a minimal nonempty weak compact convex invariant subset which contains an approximate fixed point sequence $\left(a_{n}\right)$ with $a_{n} \stackrel{w}{\longrightarrow} 0$ and, by the Goebel-Karlovitz lemma, $\lim _{n}\left\|x-a_{n}\right\|_{1}=\operatorname{diam}_{1} K=1$ for all $x \in K$.

Let $\left[X_{1}\right]:=\ell_{\infty}\left(X_{1}\right) / c_{0}\left(X_{1}\right)$ with the quotient norm given canonically by $\left\|\left[x_{n}\right]\right\|=$ $\lim \sup _{n}\left\|x_{n}\right\|_{1}$. Let $[K]:=\left\{\left[x_{n}\right]: x_{n} \in K\right.$, for $\left.n=1,2, \cdots\right\}$. Then $[T]\left[x_{n}\right]:=$ $\left[T x_{n}\right]$ is a well defined nonexpansive self mapping of $[K]$.

Given $\epsilon \in(0,1 / 2)$ let

$$
W:=\left\{\left[w_{n}\right] \in[K]:\left\|\left[w_{n}\right]-\left[a_{n}\right]\right\| \leq \frac{1}{2}-\epsilon \text { and } D\left[w_{n}\right] \leq \frac{1}{2}+\epsilon\right\},
$$

where $D\left[w_{n}\right]:=D_{1}\left(w_{n}\right)=\lim \sup _{m} \lim \sup _{n}\left\|w_{m}-w_{n}\right\|_{1}$ is well defined, since for $\left(w_{n}-y_{n}\right) \in c_{0}\left(X_{1}\right)$ we have $D_{1}\left(w_{n}\right)=D_{1}\left(y_{n}\right)$.

Then $W$ is $[T]$ invariant, closed, convex and nonempty, as $\left(\frac{1}{2}+\epsilon\right)\left[a_{n}\right] \in W$. Thus, by Lin's [8] extension of the Goebel-Karlovitz lemma, $W$ contains elements of norm arbitrarily close to one.

On the other hand, for $\left[w_{n}\right] \in W$ we may without loss of generality suppose that $w_{n} \in K$, for all $n$, and we may extract a subsequence $\left(w_{n_{k}}\right)$ such that

$\lim _{k}\left\|w_{n_{k}}\right\|_{1}=\left\|\left[w_{n}\right]\right\|$,

$\left(w_{n_{k}}\right)$ is weakly convergent to some $w_{0} \in K$, and

$d:=\lim _{k}\left\|w_{n_{k}}-w_{0}\right\|_{0}$ exists.

Now, $\left\|w_{0}\right\|_{1} \leq \liminf _{k}\left\|w_{n_{k}}-a_{n_{k}}\right\|_{1} \leq\left\|\left[w_{n}\right]-\left[a_{n}\right]\right\| \leq \frac{1}{2}-\epsilon$. Thus, given any $\eta \in(0, \epsilon)$, if $\liminf _{k}\left\|w_{n_{k}}-w_{0}\right\|_{1}<\frac{1}{2}+\eta$ we have

$$
\left\|\left[w_{n}\right]\right\| \leq \liminf _{k}\left\|w_{n_{k}}-w_{0}\right\|_{1}+\left\|w_{0}\right\|_{1}<1+\eta-\epsilon .
$$

So in this case $\left\|\left[w_{n}\right]\right\|_{1}$ is uniformly bounded away from one.

Thus, we need only consider the case when

$$
\underset{k}{\liminf }\left\|w_{n_{k}}-w_{0}\right\|_{1} \geq \frac{1}{2}+\eta .
$$

For this case, provided $b \leq\left(\frac{1}{2}+\eta\right) /\left(\frac{1}{2}-\epsilon\right)$ we have

$$
\begin{aligned}
d=\lim _{k}\left\|w_{n_{k}}-w_{0}\right\|_{0} & \geq(1 / b) \underset{k}{\liminf }\left\|w_{n_{k}}-w_{0}\right\|_{1} \\
& \geq\left(\frac{1}{2}+\eta\right) / b \\
& \geq \frac{1}{2}-\epsilon \\
& \geq\left\|w_{0}\right\|_{1} \\
& \geq\left\|w_{0}\right\|_{0} .
\end{aligned}
$$


Let $y_{k}:=(1 / d)\left(w_{0}-w_{n_{k}}\right)$, so $\left\|y_{k}\right\|_{0} \rightarrow 1$. Then

$$
\begin{aligned}
\left\|\left[w_{n}\right]\right\|=\lim _{k}\left\|w_{n_{k}}\right\|_{1} & \leq b d \limsup _{k}\left\|\frac{1}{d} w_{0}-\frac{1}{d}\left(w_{0}-w_{n_{k}}\right)\right\|_{0} \\
& =b d \psi_{\left(y_{k}\right)}\left((1 / d) w_{0}\right) \\
& \leq b d \lambda_{\left(y_{k}\right)}, \quad \text { as }\left\|(1 / d) w_{0}\right\|_{0} \leq 1, \\
& =b d D_{0}\left(y_{k}\right), \quad \text { by Lemma } 3.1, \text { as } \psi_{\left(y_{k}\right)}(0)=1, \\
& =b D_{0}\left(w_{n_{k}}\right) \leq b D_{0}\left(w_{n}\right) \leq b D\left[w_{n}\right] \leq b\left(\frac{1}{2}+\epsilon\right) .
\end{aligned}
$$

Thus again $\left\|\left[w_{n}\right]\right\|$ is uniformly bounded away from one provided $b<1 /\left(\frac{1}{2}+\epsilon\right)$.

In this way we arrive at a contradiction whenever

$$
b<\min \left(\frac{\frac{1}{2}+\eta}{\frac{1}{2}-\epsilon}, \frac{1}{\frac{1}{2}+\epsilon}\right),
$$

where $0<\eta<\epsilon<1 / 2$; that is, for $b<(1+\sqrt{5}) / 2$.

Corollary 3.3. Let $X$ be a Banach space with property (M). If $Y$ is a Banach space for which the Banach-Mazur distance $d(Y, X)<(1+\sqrt{5}) / 2$, then $Y$ has the $w-f p p$.

Proof. This follows directly from Theorem 3.2 and the observation that both the $\mathrm{w}$-fppand property $(\mathrm{M})$ are preserved if the norm is replaced by a strictly positive scalar multiple of itself.

Remarks. (1) The constant $(1+\sqrt{5}) / 2$ is equal to that obtained by Jiménez-Melado and Lloréns-Fuster [4] for $X=\ell_{2}$, and appears to be the best known for the $\ell_{p}$ spaces with $p$ near 2 .

(2) Theorem 3.2 affords another proof that $c_{0}$, while failing to have weak normal structure, none-the-less enjoys the $\mathrm{w}-\mathrm{fpp}$. Indeed in the presence of property $(\mathrm{M})$ the appearance of $c_{0}$ in the space appears to be the main impediment to weak normal structure. To see this, suppose $X$ has $(\mathrm{M})$, but fails to have weak normal structure. Since weak normal structure is separably determined, by passing to a subspace if necessary we may assume that $X$ is separable. Then, by the argument for Proposition 2.3, $X$ admits a nontrivial weakly null type

$$
\psi_{\left(x_{n}\right)}(x):=\lim _{n}\left\|x-x_{n}\right\| \quad\left(x_{n} \stackrel{w}{\longrightarrow} 0,\left\|x_{n}\right\|=1\right)
$$

which is constant on $B_{X}$. From Karlton [5] Lemma 3.6 and the discussion preceding it there exist $\left(y_{n}\right) \subset X$, a basic subsequence of $\left(x_{n}\right)$, and constants $k, K>0$ such that

$$
k\left\|\sum \xi_{n} y_{n}\right\| \leq\left\|\left(\xi_{n}\right)\right\|_{F} \leq K\left\|\sum \xi_{n} y_{n}\right\|,
$$

for all finitely supported sequences $\left(\xi_{n}\right)$, where $\|\cdot\|_{F}$ is the Orlicz norm arising from $F(t):=\lim _{n}\left\|x-t x_{n}\right\|-1$ whenever $\|x\|=1$. But, for $t \geq 1$ we readily see that $F(t)=t-1$, in particular $F$ is degenerate, so

$$
k\left\|\sum \xi_{n} y_{n}\right\| \leq\left\|\left(\xi_{n}\right)\right\|_{\infty} \leq 2 K\left\|\sum \xi_{n} y_{n}\right\|
$$

and $c_{0} \hookrightarrow X$.

Since the inclusion of $c_{0}$ is an isomorphic (and hence almost isometric) one, this does not provide a characterization of weak normal structure in spaces with property (M). It does however give an alternative, albeit substantially less direct, proof 
for some of the necessary conditions for weak normal structure given in Corollary 2.5, in particular conditions (ii) and (iii). Whether or not it captures (i) and most importantly (v), or the result of Theorem 2.4, is unclear, and leads to the question: does the Kadek-Klee property in the presence of (M) imply $c_{0} \hookrightarrow X$ ? In particular, since $c_{0}$ itself admits an equivalent Kadec-Klee norm we ask is there such a renorming which also retains property $(\mathrm{M})$ ?

(3) A dual property to $(\mathrm{M})$, property $\left(\mathrm{M}^{*}\right)$, is defined in $X^{*}$ by requiring that

$$
\psi_{\left(f_{n}\right)}: X^{*} \longrightarrow \mathbf{R}^{+}: f \longmapsto \limsup _{n}\left\|f-f_{n}\right\|
$$

be a function of $\|f\|$ only, whenever $f_{n} \stackrel{w^{*}}{\longrightarrow} 0$. Kalton [5] shows that if $X^{*}$ has $\left(\mathrm{M}^{*}\right)$ then $X$ has (M) and the natural embedding of $X$ is an M-ideal in $X^{* *}$ and so by Lima [7] $X^{*}$ has the Radon-Nikodym property. Thus, if $X^{*}$ has property $\left(\mathrm{M}^{*}\right)$, then $X^{*}$ has weak* normal structure and $X$ has the w-fpp.

(4) Since property (M) implies WORTH which in turn implies the non-strict Opial condition we are left with the question: does WORTH, or indeed the nonstrict Opial condition, imply the $\mathrm{w}-\mathrm{fpp}$ ?

We wish to thank T. Dalby for his valuable comments on early drafts of this material and in particular for suggesting the substance of Remark 2.

\section{REFERENCES}

1. van Dulst, D. and Sims, B., [1983], Fixed points of nonexpansive mappings and Chebyshev centers in Banach spaces with norms of type (KK), Proceedings of the first Romanian-GDR seminar on Banach space theory and its applications - Bucharest 1981, Springer-Verlag Lecture Notes in Mathematics 991, 35-43. MR 84i:46027

2. Edgar, G. A. and Wheeler, R. F., [1984], Topological properties of Banach spaces, Pacific J. Math. 115, 317-350. MR 86e:46013

3. Goebel, K. and Kirk, W. A., [1990], Topics in metric fixed point theory, Cambridge Studies in advanced mathematics 28, Cambridge University Press, pp244. MR 92c: 47070

4. Jiménez-Melado, A. and Lloréns-Fuster, E., [1992], Stability of the fixed point property for nonexpansive mappings, Houston J. Math. 18, 251-257. MR 93d:47097

5. Kalton, N. J., [1993], M-ideals of compact operators, Illinois J. of Math. 37, 147-169. MR 94b: 46028

6. Kalton, N. J. and Werner D., [1993], Property (M), M-ideals and almost isometric structure of Banach spaces, preprint, pp47.

7. Lima, A., [1982], On M-ideals and best approximation, Indiana Univ. Math. J. 31, 27-36. MR 83b:46021

8. Lin, Pei-Kee, [1985], Unconditional bases and fixed points of nonexpansive mappings, Pacific J. Math. 116, 69-76. MR 86c:47075

9. Maurey, B., [1980], Points fixes des contractions de certains faiblement compacts de $L^{1}$, Seminaire d'Analyse Fonctionnelle, Exposé No. VIII, pp18 MR 83h:47041

10. Sims, B., [1982], Fixed points of nonexpansive maps on weak and weak* compact convex sets, Queen's University seminar notes, pp34.

11. Sims, B., [1988], Orthogonality and fixed points of nonexpansive maps, Proc. Centre for Math. Anal., 20, Australian National University, 178-186. MR 90i:46045

12. Sims, B., [1992], The weak* Karlovitz lemma for dual lattices, Bull. Austral. Math. Soc. 45, 171-176. MR 92k:47105

Departament d'Anàlisi Matematica, Facultat de Matematiques, Universitat de València, Doctor Moliner 50, 46100 Burjassot, Spain

E-mail address: Jesus.Garcia@uv.es

Department of Mathematics, The University of Newcastle, New South Wales 2308, Australia

E-mail address: bsims@frey.newcastle.edu.au 